\title{
Consistent discretization and loop quantum geometry
}

\author{
Rodolfo Gambini ${ }^{1}$, and Jorge Pullin ${ }^{2}$ \\ 1. Instituto de Física, Facultad de Ciencias, Universidad de la República \\ Iguá esq. Mataojo, CP 11400 Montevideo, Uruguay \\ 2. Department of Physics and Astronomy, Louisiana State University, Baton Rouge, LA 70803-4001
}

(September 14th 2004)

\begin{abstract}
We apply the "consistent discretization" approach to general relativity leaving the spatial slices continuous. The resulting theory is free of the diffeomorphism and Hamiltonian constraints, but one can impose the diffeomorphism constraint to reduce its space of solutions and the constraint is preserved exactly under the discrete evolution. One ends up with a theory that has as physical space what is usually considered the kinematical space of loop quantum geometry, given by diffeomorphism invariant spin networks endowed with appropriate rigorously defined diffeomorphism invariant measures and inner products. The dynamics can be implemented as a unitary transformation and the problem of time explicitly solved or at least reduced to as a numerical problem. We exhibit the technique explicitly in $2+1$ dimensional gravity.
\end{abstract}

One of the central problems generated by the application of the rules of quantum mechanics to general relativity is the problem of the dynamics. When formulated canonically, general relativity has a vanishing Hamiltonian, which has to be implemented as a constraint. In the quantum geometry approach based on loop quantum gravity [1] the constraint has been implemented [2], but to characterize the resulting theory in a way in which the dynamics of general relativity is explicit remains a challenge. We have recently introduced a discrete approach to general relativity $[3,4]$ in which one approximates the continuum theory by a discrete theory that is constraint free. This allows to make explicit progress in the problem of the dynamics [5]. One can formulate the quantum theory in such a way that one chooses a physical variable as a clock and describes the physics relationally in terms of conditional probabilities [6]. The approach however appears radically different from usual loop quantum gravity, and does not seem to incorporate due to the discreteness, many of the attractive mathematical structures that have been developed in loop quantum gravity. In particular the characterization of states in terms of knot invariants and the existence of a rigorous mathematical arena to describe the theory.

In this paper we would like to bridge the gap between these two approaches. We will analyze the consequences of applying our consistent discretization technique to general relativity in the time-like direction, while keeping the spatial slices continuous. The resulting canonical theory has discrete time evolution and is free of constraints, as is usually the case in consistent discretizations. One can however further restrict the dynamics of the theory by imposing the diffeomorphism constraint of the usual continuum theory. Remarkably, the generator of diffeomorphisms of the continuum theory is preserved by the discrete evolution. This, in fact, is the key observation of this paper. If one starts from a formulation of general relativity based on Ashtekar's variables and performs this construction, one would have a theory that could be quantized using the usual tools of loop quantum gravity. In particular the states will be functions of spin networks that are annihilated by the diffeomorphism constraint and one can introduce the Ashtekar-Isham-Lewandowski measure and theory of integration. The usual well defined quantum operators like the area and the volume will exist and be well defined, except that in the discrete theory the total volume of the slice will be an observable since there are no further constraints. The resulting theory could be used as a basis to construct a relational quantization a la Page-Wootters [7] and introduce a physical clock that defines evolution through relational probabilities. One therefore has a mathematically well defined arena in which to complete the quantization of general relativity through a well defined procedure that can be carried out entirely, the only challenge left for completing the construction of the quantum theory being of computational nature. Of course, there is still the issue of if the resulting theory will have a correct semiclassical limit.

It may be argued that discretizing the temporal evolution while keeping space continuous is unnatural. After all space and time are supposed to be treated in the same footing in general relativity. However, it should be noted that although one starts from a spatially continuous classical theory, the loop quantization naturally introduces a discrete structure in space. Therefore the final theory will end up with both space and time discrete.

Let us illustrate the idea in the case of a $1+1$-dimensional theory in the continuum with an action $S=$ $\int d t d x L\left(q(x), \dot{q}(x), q^{\prime}(x), q^{\prime \prime}(x)\right)$ where to simplify notation we are considering only one variable and use the primes to denote derivatives with respect to the spatial coordinate. One then discretizes time and the action becomes $S=\sum_{n} L(n, n+1)$ where $L(n, n+1)$ is obtained from the Lagrangian replacing the time derivatives by $\dot{q}=\left(q_{n+1}-q_{n}\right) / \epsilon$. We assume the action has the form of a first order theory with constraints,

$$
L(n, n+1)=\int d x \pi_{n}(x)\left(q_{n+1}(x)-q_{n}(x)\right)-\epsilon \int d x H\left(q_{n}(x), \pi_{n}(x)\right)-\int d x N_{n}(x) \phi\left(q_{n}(x), \pi_{n}(x)\right)
$$


where we have assumed that the theory may have a Hamiltonian $H$ (in the case of general relativity $H$ vanishes and the theory loses all information about the discretization step $\epsilon)$ and constraint $(\mathrm{s}) \phi\left(q_{n}(x), \pi_{n}(x)\right)$. To simplify notation we are not making explicit the dependence of the Hamiltonian and the constraints on spatial derivatives of the fields, but this is allowed in our approach. One now introduces the canonically conjugate variables,

$$
\begin{aligned}
P_{n+1}(y) & \equiv \frac{\delta L(n, n+1)}{\delta q_{n+1}(y)}=\pi_{n}(y), \quad P_{n+1}^{\pi}(y) \equiv \frac{\delta L(n, n+1)}{\delta \pi_{n+1}(y)}=0, \quad P_{n+1}^{N}(y) \equiv \frac{\delta L(n, n+1)}{\delta N_{n+1}(y)}=0, \\
P_{n}(y) & \equiv-\frac{\delta L(n, n+1)}{\delta q_{n}(y)}=\pi_{n}(y)-\frac{\delta}{\delta q_{n}(y)}\left[\epsilon \int d x H\left(q_{n}(x), \pi_{n}(x)\right)+\int d x N_{n}(x) \phi\left(q_{n}(x), \pi_{n}(x)\right)\right] \\
P_{n}^{\pi}(y) & \equiv-\frac{\delta L(n, n+1)}{\delta \pi_{n}(y)}=-\left(q_{n+1}(y)-q_{n}(y)\right)-\frac{\delta}{\delta \pi_{n}(y)}\left[\epsilon \int d x H\left(q_{n}(x), \pi_{n}(x)\right)+\int d x N_{n}(x) \phi\left(q_{n}(x), \pi_{n}(x)\right)\right], \\
P_{n}^{N}(y) & \equiv \frac{\delta L(n, n+1)}{\delta N_{n}(y)}=\phi\left(q_{n}(y), \pi_{n}(y)\right) .
\end{aligned}
$$

One now can eliminate the variable $\pi$ and its canonical momentum $P^{\pi}$ and end up with a theory entirely given in terms of $q$ and its canonically conjugate momentum $P$. The theory is constraint free since equation (5) now becomes $\phi\left(q_{n}(x), P_{n+1}(x)\right)=0$ and therefore it is not a constraint, in the sense that it does not constrain variables at the same time level. If one now substitutes $P_{n+1}(x)$ making use of (3) one gets a differential equation that determines the Lagrange multiplier $N_{n}(x)$. That is, we have a theory that is constraint free at the expense of determining the Lagrange multipliers, as is usually the case in the consistent discretization approach. The resulting theory has more degrees of freedom than the continuum theory it attempts to approximate. This is due to the fact that a single solution of the continuum theory can be approximated by several, different, solutions to the discrete theory. We will now turn our attention to the specific case of general relativity and proceed to reduce the extra number of degrees of freedom by choosing a sector of solutions of the discrete theory that is preserved upon evolution. The sector is chosen by requiring that the usual diffeomorphism constraint of general relativity be satisfied. It might appear surprising at first that the requirement that the constraint be satisfied is preserved by the discrete evolution.

In order to see this, let us consider the action for general relativity written in terms of Ashtekar's variables [8],

$$
S=\int d t d^{3} x\left(\tilde{P}_{i}^{a} F_{0 a}^{i}-N^{a} C_{a}-N C\right)
$$

where $N, N^{a}$ are Lagrange multipliers, $\tilde{P}_{i}^{a}$ are densitized triads, and the diffeomorphism and Hamiltonian constraints are given by,

$$
\begin{aligned}
C^{a} & =\tilde{P}_{i}^{a} F_{a b}^{i} \\
C & =\frac{\tilde{P}_{i}^{a} \tilde{P}_{j}^{b}}{\sqrt{\operatorname{det} q}}\left(\epsilon^{i j k} F_{a b}^{i}-\left(1+\beta^{2}\right) K_{[a}^{i} K_{b]}^{j}\right)
\end{aligned}
$$

where $\beta K_{a}^{i} \equiv \Gamma_{a}^{i}-A_{a}^{i}$ and $\Gamma_{a}^{i}$ is the spin connection compatible with the triad, and $q$ is the three metric. We now proceed to discretize time. The action now reads,

$$
\begin{aligned}
S= & \int d t d^{3} x\left[\operatorname{Tr}\left(\tilde{P}^{a}\left(A_{a}(x)-V(x) A_{n+1, a}(x) V^{-1}(x)+\partial_{a}(V(x)) V^{-1}(x)\right)\right)\right. \\
& \left.-N^{a} C_{a}-N C+\mu \sqrt{\operatorname{det}} q \operatorname{Tr}\left(V(x) V^{\dagger}(x)-1\right)\right]
\end{aligned}
$$

In the above expression $V(x)=V_{I} T^{I}$ is the parallel transport matrix along a time-like direction and $F_{0 a}$ is approximated by the holonomy along a plaquette that is finite in the "time-like" direction and infinitesimal in the "space-like" direction and $T^{0}=1 / \sqrt{2}$ and $T^{a}=-i \sigma^{a} / \sqrt{2}, a=1 . .3$ and $\sigma^{\prime}$ 's are the Pauli matrices and the coefficients $V_{I}$ are real. We have omitted the subscript $n$ to simplify the notation and kept it in the quantities that are evaluated at $n+1$. The last term involves a Lagrange multiplier $\mu$ and is present in order to enforce the fact that the parallel transport matrices are unitary. We notice that the $S U(2)$ gauge invariance is preserved in the semi-discrete theory. This in turn implies that Gauss' law for the momentum canonically conjugate to the connection, $\tilde{E}_{n+1}^{a} \equiv V^{-1} \tilde{P}^{a} V$ is preserved automatically upon evolution. We do not work this out explicitly here for reasons of space, the reader can refer to the example of BF theory we present later in this paper to see how the conservation works in detail, the mechanism is similar to the one in general relativity.

We now consider a spatial (time independent) infinitesimal transformation $x^{\prime a}=x^{a}+v^{a}(x)$. It is immediate to see that the action is invariant. All variables transform as they do in the continuum action, and $V(x)$ transforms as 
a scalar. The only question could be the first term, but since the transformation is time independent the terms at $n+1$ and $n$ transform appropriately. Applying Noether's theorem, there is a resulting conserved quantity that can be readily computed using the Lagrange equations, and the resulting quantity is $C_{a}=\tilde{E}_{i}^{b} F_{a b}^{i}$ that is, the diffeomorphism constraint of the continuum theory. We have checked the conservation explicitly.

Let us outline how would one complete the quantization. The central element is to implement the canonical transformation that evolves the variables from $n$ to $n+1$ as a unitary operator. Quantum states will be functions of the connection $\Psi[A]$ that are invariant under diffeomorphisms and gauge transformations. For example one could consider cylindrical functions based on spin networks. We need to construct the unitary operator $\Psi_{n+1}\left[A^{\prime}\right]=\int D A U\left(A^{\prime}, A\right) \Psi_{n}[A]$. Since the canonical transformation that implements the evolution is generated by the Lagrangian, the unitary operator in the configuration basis is given by the exponential of the Lagrangian $[9,4]$ viewed as function of $A_{n}$ and $A_{n+1}$. In practice to compute the Lagrangian as a function of these variables one needs to solve the equations of motion between $n$ and $n+1$. In situations of interest this could be achieved numerically, for instance, or through other approximation schemes. To make the calculation feasible numerically one will have to choose to work in a subspace of states to make computations finite. The calculations in situations of great generality will be hard, but the point to emphasize here is that there is no conceptual obstacle to carrying them out. In other words, what we have here is a concrete proposal for doing numerical quantum gravity.

A point to be noted is that the calculation of the unitary evolution operator can be carried out in a context that is not diffeomorphism invariant. Given that the canonical transformation preserves the diffeomorphism generator, one has for the unitary operator that $V_{\phi} U\left(A^{\prime}, A\right) V_{\phi}^{\dagger}=U\left(\phi A^{\prime}, \phi A\right)$ where $V_{\phi}$ is the generator of a finite diffeomorphism $\phi$. If one starts from wavefunctions that are invariant under diffeomorphisms, i.e., $V_{\phi} \Psi[A]=\Psi[A]$, then one has that $U\left(A^{\prime}, A\right)$ has to satisfy,

$$
\begin{aligned}
\Psi_{n+1}\left[A^{\prime}\right] & =\int D A U\left[A^{\prime}, A\right] \Psi_{n}[A]=\int D A U\left[A^{\prime}, A\right] V_{\phi} \Psi_{n}[A]=\int D A U\left[A^{\prime}, \phi^{-1} A\right] \Psi_{n}[A] \\
& =\int D A U\left[\phi A^{\prime}, A\right] \Psi_{n}[A]=\Psi_{n+1}\left[\phi A^{\prime}\right]
\end{aligned}
$$

and the integrals can be rigorously defined using the Ashtekar-Lewandowski integration theory developed on cylindrical functions and their Cauchy completions [1].

Therefore the evolution yields a diffeomorphism invariant state. A similar comment applies to the invariance under Gauss' law (gauge invariance).

Once one has the explicit evolution of the wavefunctions, then one can choose a physical time from among the variables of the problem and construct a relational quantum theory as outlined in $[5,6]$.

It is clear that carrying out the proposal in detail in situations of interest with local degrees of freedom will require significant computational effort, even in simplified examples like the Gowdy cosmologies. To present a concrete illustration of the technique in a non-trivial setting that allows to implement things in detail we will discuss $2+1$ dimensional $S U(2)$ BF theory and see that the approach yields the correct expected results. This is of some interest since this theory is equivalent to Euclidean general relativity in $2+1$ dimensions.

We start with the standard action for BF theory $S=\int d^{3} x \operatorname{Tr}(B \wedge F)$ and we discretize the "temporal" direction (we label the "spatial" directions 1,2 and the temporal one 0$), S=\sum_{n} \int d^{2} x L(n, n+1)$ with,

$$
\begin{aligned}
L(n, n+1)= & \operatorname{Tr}\left\{B_{0}(x) F_{12}(x)+B_{1}(x)\left(A_{2}(x)-V(x) A_{n+1,2}(x) V^{-1}(x)+\partial_{2}(V(x)) V^{-1}(x)\right)\right. \\
& \left.+B_{2}(x)\left(V(x) A_{n+1,1}(x) V^{-1}(x)+V(x) \partial_{1} V^{-1}(x)-A_{1}(x)\right)+\mu\left(V(x) V^{\dagger}(x)-1\right)\right\}
\end{aligned}
$$

and we are using the same notation as in the general relativity case. In two of the three terms in the action we have approximated the curvature by a holonomy along a plaquette that is finite in the "time-like" direction and infinitesimal in the "space-like" direction. As before, the $S U(2)$ gauge invariance is preserved in the semi-discrete theory.

We now build the canonical theory as is usually done in consistent discretizations by defining the canonical conjugate momenta. All of them vanish except the conjugates to the components 1,2 of the connection, which we suggestively call $E^{1,2}$ and are given by,

$$
E^{i}(x)_{n+1}=V^{-1}(x) B^{i} V(x), \quad i=1,2,
$$

and $B^{i}=\epsilon^{i j} B_{j}$.

The definition of the canonical momenta to the $B_{n}^{i}$ 's yields evolution equations for the $A_{i}$ 's,

$$
\begin{aligned}
& P^{B^{1}}=0=A_{2}(x)-V A_{n+1,2}(x) V^{-1}(x)+\partial_{2}(V(x)) V(x)^{-1}, \\
& P^{B^{2}}=0=-A_{1}(x)+V A_{n+1,1}(x) V^{-1}(x)-\partial_{1}(V(x)) V(x)^{-1} .
\end{aligned}
$$


The momentum of $B^{0}$ vanishes and yields as equation the constraint $F_{12}=0$ and the momentum of $V$, called $P_{n+1}^{V}$ also vanishes and this yields Gauss' law $P_{n}^{V} V_{n} \equiv D_{n+1, a} E_{n+1}^{a}=0$. The constraint implies that the connection is pure gauge and the evolution equations for the connection guarantee that if one starts from a connection that is pure gauge it evolves into a pure gauge connection. We are omitting other evolution equations (like the ones for the momenta) for reasons of space.

We can outline the quantization. We choose a connection representation in which $A$ is multiplicative and $E$ is a functional derivative and they have canonical commutation relations. The evolution equations become operatorial equations among operators in a Heisenberg-like representation where the role of the ordinary time would be played by the variable $n$. If one wishes to construct the unitary transformation that would implement the dynamics in the quantum theory, one proceeds in the following way. One computes the expectation value of the equations of motion between eigenstates of the operator $A$ at instants $n+1$ and $n$. This allows to infer, by solving the resulting functional equations that the value of

$$
\begin{aligned}
U\left(A^{\prime}, A\right)= & <A^{\prime}, n+1 \mid A, n> \\
& =\delta\left(A_{1}^{\prime}-V^{-1} A_{1} V+\partial_{1}\left(V^{-1}\right) V\right) \delta\left(A_{2}^{\prime}-V^{-1} A_{2} V+\partial_{2}\left(V^{-1}\right) V\right) \exp \left(\operatorname{Tr} \int B_{0} F\right)
\end{aligned}
$$

and this expression satisfies equation (10).

Since one has constraints one needs to impose them on the space of states, and this corresponds to the usual space of states of BF theory as discussed by Ooguri and more recently by Noui and Perez [10]. As usual for BF theories, the constraints imply that the theory is spatially diffeomorphism invariant. Notice that the unitary transformation (15) depends on two free functions, $V$ and $B_{0}$. However, on the physical space these functions can be freely chosen without affecting the evolution, as can be seen by inspection of (15).

Summarizing, we have observed that one can introduce the "consistent discretization" technique in general relativity and other constrained theories in which one keeps spatial slices continuous and discretizes time. The resulting semidiscrete theory has constraints that can be solved by going to the loop representation, and therefore can be explicitly handled without conceptual problems but one can consistently impose on its space of states the diffeomorphism constraint as a further restriction. One ends up with a theory that has as physical space the usual diffeomorphism invariant kinematical structure of loop quantum gravity and one can take advantage of the various mathematical developments and physical results of that arena.

The idea that we consider continuous space and discrete time within the consistent approach was suggested to us by Lee Smolin. This work was supported in part by grants nsf-phy0244335, and by funds of the Horace C. Hearne Jr. Institute for Theoretical Physics.

[1] For recent reviews see A. Ashtekar, J. Lewandowski, Class. Quant. Grav. 21, R53 (2004) [arXiv:gr-qc/0404018]; C. Rovelli, http:/www.cp.univ-mrs.fr/ rovelli/rovelli.html; L. Smolin, [arXiv:arXiv:hep-th/0408048], T. Thiemann [arXiv:grqc/0110034].

[2] T. Thiemann, Class. Quan. Grav. 15 839; 875; 1207; 1249; 1281; 1463 (1998).

[3] R. Gambini, J. Pullin, Phys. Rev. Lett. 90, 021301 (2003) [arXiv:gr-qc/0206055]; C. Di Bartolo, R. Gambini, J. Pullin, Classical and Quantum Gravity 19, 5475 (2002) [arXiv:gr-qc/0205123].

[4] R. Gambini and J. Pullin, Class. Quant. Grav. 20, 3341 (2003) [arXiv:gr-qc/0212033].

[5] R. Gambini, R. A. Porto and J. Pullin, "Discrete quantum gravity solution to the problem of time: a model" in "Recent developments in gravity: proceedings of the 10th Hellenic Relativity Conference", World Scientific, Singapore (2003) [arXiv:gr-qc/0302064].

[6] R. Gambini, R. A. Porto and J. Pullin, Class. Quant. Grav. 21, L51 (2004) [arXiv:gr-qc/0305098]; New J. Phys. 6, 45 (2004) [arXiv:gr-qc/0402118].

[7] D. N. Page and W. K. Wootters, Phys. Rev. D 27, 2885 (1983).

[8] A. Ashtekar, J. Lewandowski Class. Quant. Grav. 21, R53 (2004) [arXiv:gr-qc/0404018].

[9] P. A. M. Dirac, Z. Phys. Sow. Band 3, Heft 1 (1933), reprinted in "Selected Papers on Quantum Electrodynamics", J. Schwinger, ed., Dover, New York (1958); see also P. Ramond "Field Theory: A Modern Primer", Benjamin/Cummings, Reading, MA (1981) p 72.

[10] H. Ooguri, Nucl. Phys. B 382, 276 (1992) [arXiv:hep-th/9112072]; K. Noui and A. Perez, [arXiv:gr-qc/0402110]. 